\title{
MODELO BIOPSICOSSOCIAL COMO NORTEADOR DO RACIOCÍNIO CLÍNICO: RELATO DE EXPERIÊNCIA
}

THE BIOPSYCHOSOCIAL MODEL AS A GUIDING PRINCIPLE TO CLINICAL THINKING: EXPERIENCE REPORT

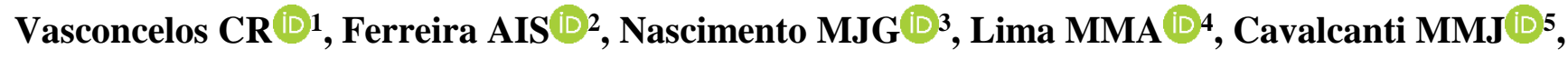 \\ Melo PVS ${ }^{10}$.
}

${ }^{1}$ Doutora em Nutrição pela Universidade Federal de Pernambuco (UFPE), Docente do Departamento de Fisioterapia, Coordenadora do Laboratório de Cinesiologia e Avaliação Funcional - LACAF, Recife, Pernambuco, Brasil. E-mail: cinthia.vasconcelos@ufpe.br. Lattes: http://lattes.cnpq.br/9932187655941209.

${ }^{2}$ Acadêmica do $5^{\circ}$ período do curso de Fisioterapia da UFPE, Recife, Pernambuco, Brasil. E-mail: anaisabel3225@gmail.com. Lattes: http://lattes.cnpq.br/3718233171013514.

${ }^{3}$ Acadêmico do $6^{\circ}$ período do curso de Fisioterapia da UFPE, Recife, Pernambuco, Brasil. E-mail: maiki.nascimento@ufpe.br. Lattes: http://lattes.cnpq.br/6190004436999642.

${ }^{4}$ Acadêmica do $9^{\circ}$ período do curso de Bacharelado em Educação Física da UFPE, Recife, Pernambuco, Brasil. E-mail: mylena.aguiar@ufpe.br. Lattes: http://lattes.cnpq.br/5345738883739506.

${ }^{5}$ Acadêmica do $3^{\circ}$ período do curso de Engenharia Biomédica da UFPE, Recife, Pernambuco, Brasil. E-mail: marina.mendonca@ufpe.br. Lattes: http://lattes.cnpq.br/7985734212081939.

${ }^{6}$ Acadêmico do $4^{\circ}$ período do curso de Fisioterapia da UFPE, Recife, Pernambuco, Brasil. E-mail: pedro.vanderlei@ufpe.br. Lattes: http://lattes.cnpq.br/9684174869254511.

Correspondência: Cinthia Rodrigues de Vasconcelos. Endereço: Avenida Jornalista Anibal Fernandes, 173, Cidade Universitária, Recife, Pernambuco, Brasil, CEP: 50740-560. E-mail: cinthia.vasconcelos@ufpe.br.

\section{Recebido: $18 / 03 / 2021$ \\ Aceito: 27/04/2021}

\section{RESUMO}

Introdução: A Classificação Internacional de Funcionalidade, Incapacidade e Saúde (CIF) é baseada num modelo biopsicossocial, utilizado como ferramenta de investigação para avaliação de questões clínicas, que são úteis para o desenvolvimento de um raciocínio clínico. Objetivo: Analisar se o modelo biopsicossocial contribui para a construção do raciocínio clínico. Metodologia: Relato de experiência, construído de forma coletiva por docente, monitores e acadêmicos da Universidade Federal de Pernambuco (UFPE), a partir da aplicação de questionário a partir de Google forms. Resultados: Verificou-se que $72,4 \%$ dos participantes já tiveram contato com a CIF, sendo relatado por $55,3 \%$ destes a utilização para a construção do raciocínio clínico, além de aplicabilidade em outros aspectos relacionados à integralidade em saúde e interdisciplinaridade. Conclusão: $\mathrm{O}$ modelo biopsicossocial da CIF contribuiu para o raciocínio clínico dos participantes, no olhar de núcleo, necessitando de inovação pedagógica para a contribuição no olhar de campo.

Palavras-chaves: Classificação Internacional de Funcionalidade, Incapacidade e Saúde. Integralidade em Saúde. Práticas Interdisciplinares.

\section{ABSTRACT}

Introduction: The International Classification of Functioning, Disability and Health (ICF) is based on a biopsychosocial model applied as an investigation tool for evaluation of clinical matters, useful to developing a clinical thinking. Objective: Analysing wether the biopsychosocial model contributes to building a clinical way of thinking. Methodology: It is an experience report built collectively by professors, mentors and students from the Federal University of Pernambuco (FUPE), from answers given by participants on Google forms. Results: It was seen that $72,4 \%$ of the participants have already had contact with ICF, 55,3\% of them associated it with building a clinical way of thinking, as well as other aspects related to integration in the field of health and interdisciplinarity. Conclusion: ICF's biopsychosocial model contributed to the clinical thinking of the participants in nucleus view, nevertheless, there is still a need for pedagogical innovation contributing to the field view.

Keywords: International Classification of Functioning, Disability and Health. Integrality in Health. Interdisciplinary Placement. 


\section{INTRODUÇÃO}

De acordo com com a Lei $n^{\circ} 8.080$ de 19 de Setembro de 1990 da Constituição Federal (1990) ${ }^{1}$ também conhecida como lei orgânica de saúde, é assegurado aos cidadãos brasileiros o Princípio de Integralidade, que tem por definição o conjunto articulado e contínuo das ações e serviços preventivos e curativos, individuais e coletivos, exigidos para cada caso em todos os níveis de complexidade do sistema.

Para a integralidade no cuidado, faz-se necessário a união de linhas de conhecimentos multiprofissionais, unidas para entender o paciente de maneira biopsicossocial, visando a construção para uma transdisciplinaridade, que devem ser consideradas para a obtenção de um raciocínio clínico capaz de alcançar a resolutividade das incapacidades apresentadas pelos usuários, como já discorria Silva, Miranda e Andrade $(2017)^{2}$ ao falar que, deve haver diálogo entre as distintas profissões, visando uma abordagem mais completa e efetiva, pois essa resolubilidade garante direito este assegurado através da legislação brasileira.

Segundo o Ministério da Saúde $(2017)^{3}$ todo profissional apresenta competências específicas, visando as demandas peculiares individuais ou coletivas que deságuam na interface de cada profissional, e comuns entre si que possibilita uma maior qualidade oferecida de atenção, bem como, suas habilidades; com isso é perceptível a possibilidade da integração dos saberes. Assim, é importante a definição de olhar de campo e olhar de núcleo. O olhar de núcleo demarca a identidade de uma área de saber e de prática profissional; e o olhar de campo, por sua vez, refere-se a um espaço de limites imprecisos onde cada disciplina e profissão buscaria em outras um apoio para cumprir suas tarefas teóricas e práticas. ${ }^{4}$

Com isso, a construção do raciocínio clínico baseado no modelo biopsicossocial com um olhar tanto de núcleo quanto de campo é ganho para a saúde, corroborando para a uma conduta transdisciplinar, fortalecendo o aspecto de cuidado integral do paciente. Chegando a realidade de que o processo de saúdeenfermidade-intervenção não é monopólio nem ferramenta exclusiva de nenhuma especialidade, pertencendo a todo o campo da saúde. ${ }^{5}$

O raciocínio clínico é uma habilidade essencial em todas as áreas do conhecimento que são relacionadas à saúde ou não. Tal habilidade permite ao profissional uma noção de como proceder perante quadros clínicos adversos que o mesmo vai se deparar. Para que um profissional possa fechar um diagnóstico médico ou funcional o mesmo precisa de vários fatores, mas sem essa habilidade fica mais difícil chegar a uma conclusão assertiva e com isso o tratamento não ocorrerá de forma satisfatória. ${ }^{6}$

Por volta dos anos 2000, houve a necessidade de estabelecer um novo modelo de Classificação em saúde, que trouxesse a abordagem biopsicossocial como premissa básica para o cuidado, diferentemente do que era proposto na abordagem biomédica, proposto pela Classificação Internacional de Doenças (CID). Assim foi publicada a Classificação Internacional de Funcionalidade, Incapacidade e Saúde (CIF), em 2001, pela Organização Mundial da Saúde (OMS). ${ }^{7}$ 
Dentre as diversas aplicações da CIF, pode-se citar que a mesma pode ser utilizada como uma ferramenta de investigação (para medir resultados, a qualidade de vida ou os fatores ambientais) ou para avaliar questões clínicas (necessidades; compatibilidade de tratamentos com as condições específica; avaliação de desempenho; os resultados de processos, como os de reabilitação). Estas aplicações são úteis para o desenvolvimento de um raciocínio clínico que necessita de indicadores assistenciais para monitoramento da efetividade dos processos realizados. ${ }^{7}$

O modelo biopsicossocial que atende não apenas ao nível físico e patológico dos sinais e sintomas que estão atrelados a CID-10 (baseado apenas no processo saúde-doença), mas considera também os fatores sociais, ambientais e psicológicos do indivíduo, sendo observado de forma global, possibilitando um cuidado mais amplo e efetivo. ${ }^{8}$ Os componentes da CIF se interligam e compõem um modelo multidimensional, multidirecional e dinâmico, conforme pode ser observado na FIGURA 1.

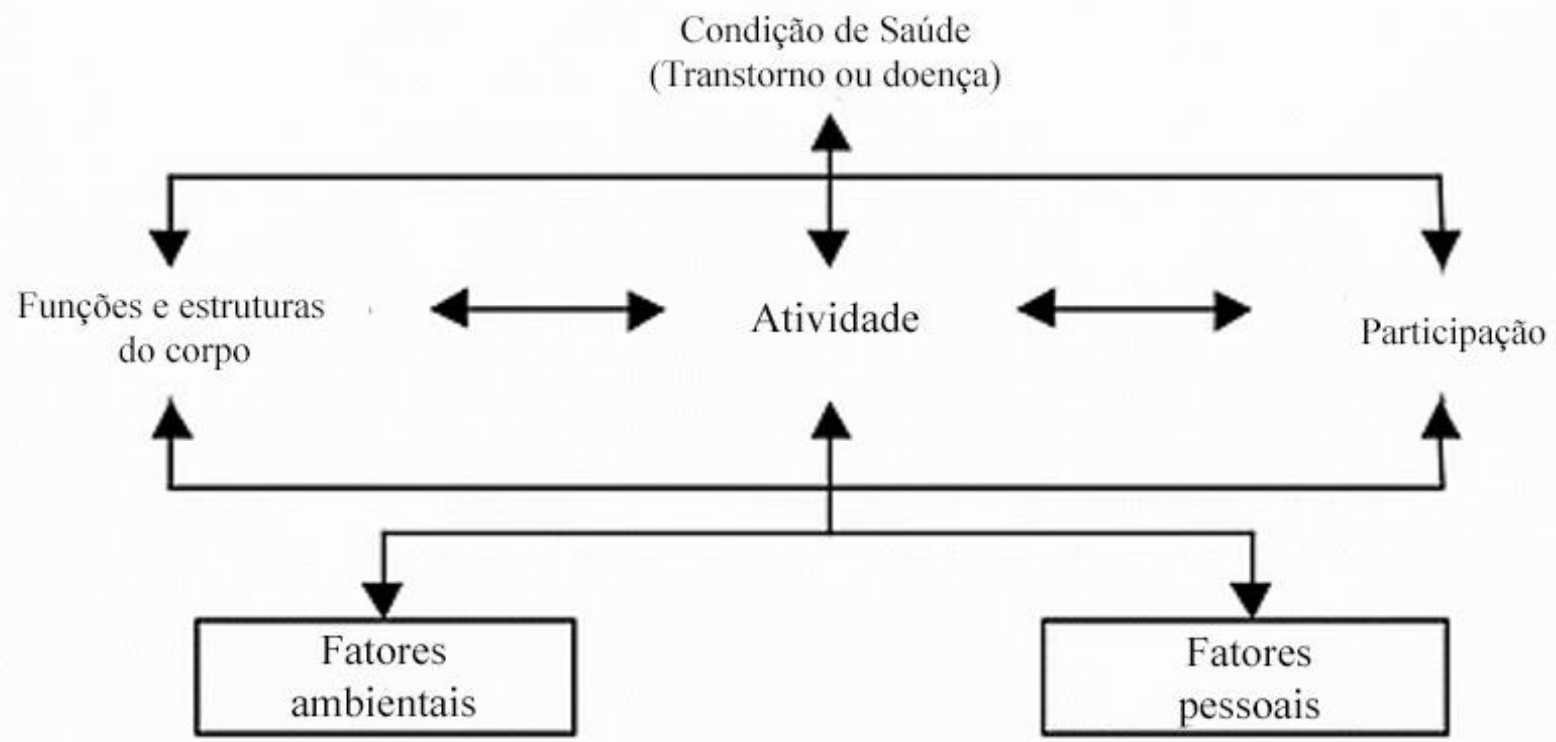

FIGURA 1 - Modelo biopsicossocial da CIF.

Fonte: Fig. 1. Interactions between the components of ICF (WHO, 2002 p.18) traduzido pelos autores.

Além do mais, ao avaliar um paciente envolvendo a CIF, dá-se margem para a análise do estado dos fatores físicos acometidos pela doença (estrutura e função do corpo), como também aos fatores pessoais e ambientais que são barreiras ou facilitadores para incluí-lo nas atividades e participações da vida diária.

Por fim, mediante considerações realizadas, o presente estudo analisou se o modelo biopsicossocial contribui para a construção de um raciocínio clínico de acadêmicos universitários, tanto no olhar de núcleo (curso de Fisioterapia) quanto no de campo (diversos cursos). 


\section{METODOLOGIA}

Trata-se de um estudo descritivo, do tipo relato de experiência, com aplicação de questionário de pesquisa considerado de opinião pública, por não possuir identificação do participante, ser de caráter pontual, e realizado por meio de metodologia específica, através da qual o participante é convidado a expressar sua preferência, avaliação ou o sentido que atribui a temas específicos. ${ }^{9}$ Nesse sentido, por se tratar de uma pesquisa que envolveu a opinião pública, a resolução do Conselho Nacional de Saúde $\mathrm{n}^{\circ}$ 510/2016, determina que não é necessária avaliação pelo Comitê de Ética em Pesquisa (CEP) / Comissão Nacional de Ética em Pesquisa - CONEP. ${ }^{10}$

Este relato surgiu a partir da inquietação de docentes e monitores sobre a necessidade de avaliar experiências acadêmicas que foram possibilitadas em disciplinas, como estratégias pedagógicas em aulas, buscando desenvolver o olhar integral e o interprofissionalismo nos acadêmicos. Sendo assim, o questionário aplicado objetivou a compreensão sobre a percepção dos acadêmicos quanto a aplicação do Modelo Biopsicossocial para o seu fazer profissional.

A pesquisa desenvolvida se deu sob responsabilidade dos autores deste trabalho $(n=6)$, envolvidos enquanto discentes e monitores, com as Disciplinas "Avaliação em Fisioterapia (ofertada apenas para acadêmicos do Curso de Fisioterapia)" e "Movimento, Funcionalidade e Saúde (ofertada para todos os cursos da Universidade)", ambas ofertadas pelo Departamento de Fisioterapia da UFPE. Quanto ao questionário aplicado, este envolveu membros da comunidade acadêmica da Universidade Federal de Pernambuco (UFPE).

Para a análise do Olhar de Núcleo, estiveram envolvidos a docente, os monitores e o estudante, todos do curso de Fisioterapia. Para o Olhar de Campo, foram compreendidos os autores do relato que já tiveram alguma participação nas atividades propostas pela disciplina "Movimento, Funcionalidade e Saúde". Além disso, participaram da pesquisa 57 estudantes de 10 cursos da UFPE do Campus Recife, a partir das respostas fornecidas pelo questionário divulgado por mídias digitais. O link para participação da pesquisa foi enviado através do e-mail institucional para todos os discentes que haviam cursado as disciplinas ou que estavam devidamente matriculados no semestre de realização da pesquisa, além do compartilhamento em grupos de WhatsApp.

A construção desse relato ocorreu durante o semestre letivo 2020.1 da UFPE, ofertado de janeiro a abril de 2021, devido à adaptação do calendário em função da suspensão das aulas presenciais em virtude da pandemia de COVID-19. Todo planejamento e execução deste relato aconteceu de forma coletiva e totalmente remota.

RELATO DA EXPERIÊNCIA DO OLHAR DE NÚCLEO:

Como este relato de experiência refere-se a uma estratégia pedagógica utilizada por duas disciplinas 
do curso de fisioterapia, que utilizou a CIF e o modelo biopsicossocial proposto pela classificação em suas aulas, julgou-se importante o conhecimento das ementas destes componentes curriculares, bem como os conteúdos programáticos e metodologias utilizadas, conforme pode ser observado no relato da experiência propriamente dito.

A disciplina "Avaliação em Fisioterapia”, do curso de Fisioterapia, com 75 horas (15 h teóricas e $60 \mathrm{~h}$ práticas) tem a seguinte Ementa:

Ementa da Disciplina Avaliação em Fisioterapia: Estuda a metodologia científica e o uso de estatísticas na análise das investigações clínicas em fisioterapia. oferece conhecimento teórico e experiência prática e clínica para avaliação de: posturas, marcha. atividades motoras básicas, teste de função muscular, teste de amplitude articular, teste de capacidade respiratória, medidas de comprimento e circunferência dos membros, avaliação do desenvolvimento motor, avaliação do sistema sensório-motor e eletrodiagnóstico da função neuro-muscular. ${ }^{11}$

Analisando a ementa, que fora construída no interstício de 2004-2007, observa-se que não há terminologias que possam ser associadas à CIF, nem ao modelo biopsicossocial, interprofissional ou mesmo à integralidade em saúde, visto que a CIF ainda estava sendo implantada pela OMS. Para amenizar esta realidade, ressalta-se que a ementa não pode ser modificada a não ser que haja uma reforma do projeto curricular do curso (PPC). O máximo de ajuste que pode acontecer é no conteúdo programático, na metodologia e nas ferramentas metodológicas utilizadas nas aulas.

Atualmente o conteúdo programático é todo voltado para a formação por competências, objetivando que o aluno seja capaz de realizar uma avaliação fisioterapêutica embasada em conhecimentos teóricos sobre funções e estruturas do corpo; atividades e participação social; fatores ambientais; além de técnicas e métodos de avaliação para as diversas especialidades da fisioterapia. As habilidades técnicas, além das comportamentais, são estimuladas em todas as aulas, especialmente nas de natureza prática.

O conteúdo programático é iniciado, semestralmente, com a conscientização da identidade profissional do fisioterapeuta, embasando-se especialmente nas legislações norteadoras da profissão. $\mathrm{O}$ acadêmico é estimulado a entender a funcionalidade humana, e qual sua relação com o controle motor (postura e movimento). A CIF já é inserida nas primeiras aulas, o que inclui também a apresentação e exploração do modelo biopsicossocial.

Com a utilização desse modelo biopsicossocial, os alunos se colocam na condição de um usuário e apresentam-se uns aos outros, falando de seus fatores pessoais, condições de saúde, atividade e participação social que tenham alguma incapacidade real e que julgam ser importante a sua revelação para a turma. As estruturas e funções do corpo envolvidos são inseridas no modelo, como também os fatores ambientais 
identificados. Nessa mesma lógica, um outro exercício aplicado é a construção desse modelo para situações clínicas possíveis de serem identificadas nas diversas especialidades da fisioterapia.

A partir daí, todos os conteúdos apresentados são sempre contextualizados na CIF. Casos clínicos são sempre trazidos à aula, para que, inclusive, a conscientização do trabalho interprofissional seja estimulada entre os alunos da fisioterapia. O raciocínio clínico é estruturado, a partir do modelo biopsicossocial, iniciando pela avaliação fisioterapêutica, seguida do diagnóstico fisioterapêutico, objetivos terapêuticos (o funcional e os respectivos objetivos estruturais), planejamento terapêutico e critérios para alta fisioterapêutica.

Uma outra metodologia utilizada na disciplina é o acrônimo SMART ( $\mathrm{S}=$ específico, $\mathrm{M}=$ mensurável, $\mathrm{A}=$ atingível, $\mathrm{R}=$ relevante, $\mathrm{T}=$ temporal) para a definição dos objetivos terapêuticos, focado na funcionalidade, dentre tantas atividades existentes para serem realizadas pelo ser humano. Respeitandose a abordagem biopsicossocial, esse olhar integral estimulado nos alunos, deve ser adequado para a necessidade do paciente, já que o cuidado é centrado no mesmo.

Alguns depoimentos trazem a percepção dos envolvidos na disciplina "Avaliação em Fisioterapia" em relação ao momento de inserção da CIF na organização da disciplina, como também à sua forma de utilização e apresentação em aulas:

"Como aluno pude vivenciar a experiência de trabalhar com a CIF logo na graduação, no $4^{o}$ período, isso me ajudou bastante com a criação de uma percepção maior com relação a avaliar e diagnosticar um paciente. A CIF te permite codificar muitos aspectos que estão ligados aos pacientes, tais como sua parte do corpo e até mesmo fatores ambientais que vão servir como facilitador ou como barreira. Isso me permite ter uma visão mais ampla, assertiva, que vai facilitar no meu diagnóstico e posteriormente na intervenção que será utilizada em cada paciente. A CIF vem para facilitar a vida dos profissionais, tanto dos acadêmicos. A mesma auxilia na construção do raciocínio clínico que foi uma das coisas que ela me proporcionou."

Aluno da disciplina de Avaliação em Fisioterapia, cursando em 2020.1.

"Como monitor da disciplina de avaliação em fisioterapia no semestre 2019.2 pude perceber a dificuldade dos alunos ao manusear a ferramenta CIF. No semestre 2020.1 houve uma reorganização da disciplina, onde trouxe o modelo biopsicossocial da CIF como ferramenta primária para a construção de aspectos como anamnese fisioterapêutica, diagnóstico principal e funcional, na construção de raciocínio clínico nas propostas de 
atividades em sala, e nos foi dado um feedback positivo perante isso, eles a partir do modelo desbravaram a CIF para codificar e qualificar os achados, conseguindo apurar melhor o olhar de campo e de núcleo a partir do modelo biopsicossocial, sabendo olhar o paciente como o todo, o colega da sala, a docente, o monitor, como pessoas que tem características, crenças, $e$ composições distintas."

Monitor da Disciplina de Avaliação em Fisioterapia, no período de 2019.2 e 2020.1

“Atualmente estou como monitora da disciplina, e percebo uma modificação na forma como a CIF foi apresentada aos alunos da minha turma, em 2019.2 e da nova turma de 2020.1. Essa mudança no cronograma da disciplina proporcionou uma diferença significativa no aproveitamento e utilização da CIF entre os alunos dos determinados grupos. Fez toda diferença! Fez com que o aluno tenha um olhar mais abrangente, que vai além da análise do estado físico patológico do usuário, mas sim, que adentra os fatores sociais e ambientais daquele indivíduo por saber que eles são arduamente importantes para limitar ou desenvolver a atividade e participação do paciente no seu dia a dia."

Monitora da Disciplina de Avaliação em Fisioterapia, em 2020.1.

\section{RELATO DA EXPERIENNCIA DO OLHAR DE CAMPO:}

A disciplina "Movimento, Funcionalidade e Saúde", ofertada pelo Departamento de Fisioterapia para todos cursos da UFPE, com 45 horas (teóricas) tem a seguinte Ementa:

Ementa da Disciplina Movimento, Funcionalidade e Saúde: Estudo do movimento humano aplicado à funcionalidade e à saúde, num olhar interprofissional. A definição da identidade profissional das diferentes categorias que utilizam o movimento humano será o elemento norteador para proporcionar aos acadêmicos competências para analisar os movimentos normais que compõem a funcionalidade humana. Conhecimentos anatômicos, fisiológicos, biomecânicos e cinesiológicos serão estudados, sob a perspectiva da funcionalidade humana. Habilidades para identificar os desvios da normalidade do movimento humano serão construídas. As questões atitudinais necessárias ao trabalho interprofissional, com garantia à integralidade em saúde, serão estimuladas a partir da abordagem 
biopsicossocial, entendendo que os fatores ambientais podem ser facilitadores ao alcance da saúde, no seu contexto mais amplo. ${ }^{11}$

No caso dessa disciplina, a ementa traz terminologias que podem ser associadas diretamente à CIF, ao modelo interprofissional e à integralidade em saúde. A construção da ementa foi feita no ano de 2020 e a criação dessa disciplina, mesmo na condição de eletiva, foi feita estrategicamente para alcançar esses princípios. A disciplina se propõe em promover condições para que os discentes matriculados adquiram conhecimentos acerca dos aspectos funcionais envolvidos nas diversas possibilidades que englobam o movimento humano, a partir de um olhar integral e interprofissional.

Essa disciplina eletiva, recém construída, está no seu segundo semestre de oferta. No primeiro deles, a oferta foi em caráter experimental, tendo 20 alunos matriculados, com os seguintes cursos de origem: Educação Física, Engenharia Biomédica, Fisioterapia e Terapia Ocupacional. Para as atividades em aula, a turma foi dividida em duas equipes, cada uma delas contendo integrantes dos quatro cursos matriculados na disciplina.

No segundo semestre de oferta, num semestre letivo tradicional, oito alunos matricularam-se, oriundos dos cursos de Educação Física, Engenharia Biomédica e Fisioterapia. Como essa experiência é nova e ainda está numa fase de implementação e adaptação, a metodologia empregada foi ajustada à realidade e expectativa da turma. Por conta do quantitativo de alunos matriculados, a turma integrou-se, de forma a compor uma única equipe/time de trabalho.

O conteúdo programático inicia-se com a conscientização da identidade profissional de cada uma das profissões referentes aos cursos de origem dos acadêmicos matriculados. Essa identidade sempre é contextualizada na importância para a abordagem integral e para o trabalho interprofissional. Assim a CIF é inserida, apresentando-se como uma solução para a organização do raciocínio clínico, onde a partir do modelo biopsicossocial, cada profissão organiza-se na perspectiva do olhar do núcleo e, posteriormente, no olhar do campo.

Para consolidar o aprendizado, a docente após algumas aulas já realizadas, solicita que os discentes utilizem o modelo biopsicossocial para se apresentarem mais intimamente, já que houve contatos prévios. A ideia é que a equipe fique mais integrada. Cada pessoa fala de seus fatores pessoais, condições de saúde, atividade e participação social. As estruturas e funções do corpo envolvidos são inseridas no modelo, como também os fatores ambientais identificados.

Outra estratégia é a utilização de casos clínicos para que cada aluno transfira os dados apresentados para o modelo biopsicossocial. Em seguida há simulação de uma discussão de caso clínico, onde todos são incentivados a argumentarem o raciocínio empregado. A partir da facilitação da docente, todos são incentivados a construir os objetivos terapêuticos que seriam adotados pela equipe. O acrônimo SMART é 
utilizado para manter o foco de todos os envolvidos.

Por volta de metade da carga horária da disciplina, um caso clínico real é apresentado à turma para que todos possam vivenciar a experiência de uma abordagem interprofissional. $O$ passo a passo para o raciocínio clínico estruturado é incentivado e cada aluno necessita buscar em seu curso um docente que possa assumir o papel de tutor para desenvolver esse projeto real, responsabilizando-se pelas especificidades de cada profissão, já que a docente da disciplina é Fisioterapeuta de formação.

Nessa fase da disciplina é que a ferramenta 5W2H é apresentada para a equipe, com o objetivo de organizar o planejamento terapêutico e os indicadores que serão utilizados para o monitoramento dos resultados alcançados. Essa fase segue os princípios do Projeto Terapêutico Singular (PTS), utilizado principalmente pelos profissionais da atenção básica. Com o planejamento construído e amplamente discutido pela equipe, o mesmo é apresentado ao paciente para que sua ciência e adesão seja obtida.

Por fim, o projeto é apresentado em aula, a vários docentes, profissionais e acadêmicos convidados, de diversas expertises profissionais, para que haja a defesa da ideia/planejamento pela equipe e que as contribuições dos especialistas possam ser obtidas. Se for possível, a depender do contexto, o planejamento é colocado em prática, a depender das parcerias/articulações que tenham sido estabelecidas ao longo da disciplina. Com essa defesa do projeto, os acadêmicos são estimulados a desenvolver habilidades comportamentais como: comunicação eficiente, criatividade, flexibilidade cognitiva, inteligência emocional, pensamento crítico, julgamento e tomada de decisões, negociação, foco no objetivo a ser alcançado, proatividade, resolução de problemas concretos e cooperação com os outros (trabalho em equipe).

Nessa perspectiva de construção coletiva e orientação da disciplina ao processo de aprendizado da turma, feedbacks são obtidos constantemente no decorrer das aulas, conforme pode ser observado abaixo: "Ao trocar experiências pessoais e profissionais de forma constante na disciplina, acerca dos elementos presentes na identidade profissional de cada discente e seus respectivos cursos, foi possível pensar nas diversas possibilidades de complementaridade do conhecimento para a construção do raciocínio clínico. Para tal, o Modelo Biopsicossocial foi utilizado com o objetivo de promover condições para pensar o aluno ou paciente como ser integral. A experiência proporcionada na disciplina foi de suma importância para o meu processo de formação, principalmente por promover discussões acerca de temas ainda desconhecidos, a partir de um olhar interdisciplinar, e ainda por estimular o desenvolvimento de habilidades importantes para o trabalho em equipe e resolução de problemas, baseado em situações reais do contexto profissional." 
Aluna da disciplina de Movimento, Funcionalidade e Saúde, cursando em 2020.1 .

"Como estudante de Engenharia Biomédica, posso dizer que a disciplina de Movimento, Funcionalidade e Saúde abre a mente de quem tem interesse na área de reabilitação, por estar já introduzindo o estudante em assuntos mais práticos com os conceitos do Modelo Biopsicossocial e da CIF, em um caso real e em contato com profissionais e estudantes da área de saúde, como Fisioterapia, Educação Física e Terapia Ocupacional. É bem gratificante em meio a bastante carga teórica da engenharia ter uma cadeira e estudo mais prático, além de claro ajudar alguém. Na minha experiência, eu recomendo, para quem tem interesse de cursar, ter pago ou pagar simultaneamente a disciplina de Biomecânica ou de Cinesiologia, por dar base em conceitos que serão muito úteis ao longo da disciplina."

Aluna da disciplina de Movimento, Funcionalidade e Saúde, cursando em 2020.1 .

\section{RESULTADOS}

Abaixo estão os resultados da percepção de acadêmicos de diversos cursos sobre a construção do raciocínio clínico, a partir do modelo lógico da CIF.

O perfil dos 57 acadêmicos voluntários desta pesquisa está disposto na TABELA 1, sendo a maioria do sexo feminino (75,4\%), com 20 anos de idade (29,8\%), estudantes do curso de Fisioterapia $(63,1 \%)$, cursando o $4^{\circ}$ período do curso $(33,3 \%)$ e já cursaram as disciplinas relacionadas ao questionário $(63,2 \%)$.

Quanto às percepções dos alunos, dos 10 cursos envolvidos, verifica-se a seguinte realidade, conforme dados apresentados na TABELA 2. 
TABELA 1 - Perfil de acadêmicos do Campus Recife da Universidade Federal de Pernambuco, sobre a percepção da construção do fazer profissional, baseando-se nas disciplinas "Avaliação em Fisioterapia" e "Movimento, Funcionalidade e Saúde" (Abril/2021).

\begin{tabular}{|c|c|c|c|}
\hline VARIÁVEIS & & $\mathrm{n}$ & $\%$ \\
\hline \multirow{3}{*}{ SEXO } & Masculino & 13 & 22,8 \\
\hline & Feminino & 43 & 75,4 \\
\hline & Prefiro não especificar & 1 & 1,8 \\
\hline \multirow{10}{*}{ CURSO } & Biomedicina & 2 & 3,8 \\
\hline & Educação Física & 8 & 14 \\
\hline & Engenharia de Alimentos & 1 & 1,7 \\
\hline & Estatística & 1 & 1,7 \\
\hline & Fisioterapia & 36 & 63,1 \\
\hline & Geografia & 2 & 3,5 \\
\hline & Museologia & 1 & 1,7 \\
\hline & Psicologia & 2 & 3,5 \\
\hline & Sistema de Informação & 1 & 1,7 \\
\hline & Terapia Ocupacional & 3 & 5,2 \\
\hline \multirow{3}{*}{ JÁ CURSOU AS DISCIPLINAS } & Avaliação em Fisioterapia & 29 & 50,9 \\
\hline & Movimento, Funcionalidade e Saúde & 7 & 12,3 \\
\hline & Nenhuma das opções & 21 & 36,8 \\
\hline
\end{tabular}

Legenda: $n$ : amostra ( $\mathrm{n}=57) . \%$ : porcentagem.

TABELA 2 - Percepção de acadêmicos do Campus Recife da Universidade Federal de Pernambuco sobre a contribuição da CIF na formação profissional (Abril/2021).

PERGUNTAS

VOCÊ JÁ TEVE CONTATO COM A CIF?

COMO VOCÊ JÁ TEVE CONTATO COM A C
A CIF CONTRIBUIU NA SUA FORMAÇÃO
ACADÊMICA?

VOCÊ CONHECE O MODELO BIOPSICOSSOCIAL?

O MODELO BIOPSICOSSOCIAL CONTRIBUIU PARA O APRIMORAMENTO DO SEU RACIOCÍNIO CLÍNICO? RESPOSTAS $\%$

\begin{tabular}{cc} 
Sim & 72,4 \\
\hline Não & 27,6 \\
\hline Disciplinas & 92,9
\end{tabular}

Projetos de Pesquisa ou de Extensão $\quad 4,8$

Estágios 2,4

\begin{tabular}{cc}
\hline Raciocínio clínico & 55,3 \\
\hline Integralidade & 27,7 \\
\hline Interdisciplinaridade & 6,4 \\
\hline Não contribuiu em nada & 10,6 \\
\hline Sim & 63,8 \\
\hline Não & 36,2 \\
\hline Sim & 76,1 \\
\hline Não & 23,9 \\
\hline
\end{tabular}

PARA A CONSTRUÇÃO DO RACIOCÍNIO CLÍNICO NA PERSPECTIVA DA Sim 94,4

INTERDISCIPLINARIDADE É NECESSÁRIO A

PARTICIPAÇÃO DE OUTROS PROFISSIONAIS?

Legenda: $n$ : $(n=57) . \%$ : porcentagem. 


\section{DISCUSSÃO}

A percepção dos acadêmicos que cursaram as disciplinas "Avaliação em Fisioterapia" e "Movimento, Funcionalidade e Saúde" é positiva em relação à contribuição da mesma no aprimoramento do raciocínio clínico, integralidade em saúde e interprofissionalismo, já que a maioria dos participantes relataram ter cursado as mesmas. A CIF e o modelo biopsicossocial, dentro das metodologias empregadas nas aulas das duas disciplinas em questão, parecem ser um dos grandes responsáveis por esta percepção. O objetivo era promover condições aos discentes para pensar o paciente ou aluno como ser integral, levando em consideração sua condição física, atividade, participação social, fatores pessoais e ambientais. ${ }^{12}$

$\mathrm{Na}$ análise dos acadêmicos, é possível vivenciar elementos únicos do processo de ensinoaprendizagem, principalmente quando são utilizadas estratégias pedagógicas que promovam uma maior troca de experiência interpessoal e interdisciplinar, a partir da utilização de recursos didáticos e métodos cada vez mais dinâmicos e autônomos neste processo, como é o caso das metodologias ativas. ${ }^{13-14}$

As disciplinas relatadas nessa experiência utilizaram, na prática pedagógica para a apresentação e exploração do modelo biopsicossocial da CIF, os elementos das metodologias ativas, como Sala de Aula Invertida, Aprendizagem Baseada em Problemas e em Projeto. ${ }^{10}$ Outros elementos também foram experimentados para potencializar o processo de ensino-aprendizagem (como aprendizagem baseada em times); a utilização de ferramentas de gestão para o planejamento e acompanhamento de ações (5W2H); ${ }^{15}$ ferramentas de interações digitais para monitoramento de tarefas coletivas em tempo real (G-Suits e aplicativo Trello), principalmente devido ao contexto da Atividade Educacional Remota Emergencial. ${ }^{16}$

Retornando à análise do presente relato de experiência sobre o modelo biopsicossocial, defendido pela CIF, em relação à sua contribuição para a construção de um raciocínio clínico, tanto na visão de núcleo quanto na de campo, é preciso que essa hipótese seja monitorada com pesquisas de metodologia seguras e confiáveis. Estudos já apontam a CIF como sendo uma das possibilidades alternativas do cuidado em saúde, que proporciona um olhar mais amplo e humanizado. ${ }^{17} \mathrm{O}$ respeito às múltiplas dimensões envolvidas no processo de saúde e funcionalidade/incapacidade (biológica, individual, social), além do reconhecimento do importante papel do ambiente (não só físico, mas social e de atitudes), traz uma nova luz sobre "saúde" e "incapacidade". $7-8$

Entretanto, ao pensar no modelo biopsicossocial, faz-se necessário abordar a interdisciplinaridade presente na sua aplicabilidade e finalidade. Para que ocorram discussões acerca da interdisciplinaridade, é preciso que profissionais de categorias diferentes estejam envolvidas, entretanto outras formas de experiências também necessitam ser proporcionadas aos envolvidos, principalmente quando se fala no processo de formação.

A perspectiva do olhar do campo só é possível quando o olhar do núcleo é fortalecido. A identidade profissional deve ser identificada, de forma clara em termos de conceitos e atuação, sendo esta uma das 
primeiras atividades a serem propostas nas diversas disciplinas, dentro de seus contextos, como aconteceu nas disciplinas aqui estudadas. A qualidade de nossos compartilhamentos interferirá nas configurações contemporâneas do trabalho e, assim, em nossas compreensões, relacionamentos e disposição de nossos saberes-fazer. ${ }^{18}$

Edgar Morin $(1921)^{19}$ já relatava que o enfraquecimento de uma percepção global levava ao enfraquecimento do senso de responsabilidade. Ou seja, nos dias atuais, constata-se que para um olhar integral é preciso se auto conhecer (identidade profissional), perceber e conhecer o outro, pois assim é possível saber o que temos para contribuir, como também quem são os outros atores que também poderão participar desse processo. Por isso, em todo o processo de formação de RH, a convivência com categorias profissionais é imprescindível para que o conhecimento entre as especificidades dos cursos (profissões) aconteça.

Apropriar-se sobre o ser e fazer profissional é de suma importância para o processo de formação, pois permite um olhar mais abrangente e humilde sobre as possibilidades de atuação futura, pautada na responsabilidade social de cada indivíduo, seja na perspectiva da carreira acadêmica ou profissional. ${ }^{20-21}$

Nessa perspectiva, ao trocar experiências pessoais e profissionais acerca dos elementos presentes na identidade profissional de cada discente e seus respectivos cursos, fez-se a necessidade de pensar nas possibilidades de complementaridade para a construção do raciocínio clínico. Segundo Sampaio et al. ${ }^{22}$ (2005), a CIF é um modelo que auxilia o profissional a conduzir e desenvolver um plano de tratamento orientado e focado de forma individual para cada paciente.

Peduzzi $(2007)^{23}$ refere que se entende que a educação interprofissional contribui na formação de profissionais da saúde que estejam melhor preparados para o desafio que é trabalhar junto e de forma integrada na perspectiva da integralidade. Ou seja, ainda se faz necessário mais caminhos, disciplinas, projetos de pesquisas, extensões, entre tantos outros pontos que devemos trabalhar de forma integral, não só para a construção do saber, mas para uma construção pessoal e profissional. O que torna urgente que se iniciem mudanças no sistema convencional do ensino em saúde, baseando-se em uma intervenção voltada cada vez mais para a abordagem biopsicossocial ao invés do modelo biomédico.

\section{CONCLUSÃO}

O raciocínio clínico se refere ao processo cognitivo, através do qual, profissionais são capazes de ter uma tomada de decisão, a fim de estabelecer diagnóstico correto e propor uma conduta adequada frente a um problema clínico encontrado.

Esse estudo observou que o ponto de partida dos discentes, no início das disciplinas em questão, é o olhar fragmentado no cuidado em saúde, mas à medida que o modelo biopsicossocial foi apresentado e que essa abordagem avançou nas discussões, o raciocínio clínico começou a ser construído com esse novo 
olhar. Os pré conceitos inerentes à saúde foram desconstruídos. A partir daí os discentes passaram a considerar a abordagem biopsicossocial imprescindível na construção do raciocínio clínico de cada indivíduo.

Assim, conclui-se que o modelo biopsicossocial da CIF, nesta população, contribuiu para o raciocínio clínico no olhar de núcleo, devendo ainda haver mais investimentos pedagógicos para a contribuição no olhar de campo, sendo as atividades com caráter interprofissional, uma opção eficaz durante o processo de formação de profissionais de saúde a fim de garantir a integralidade no cuidado.

\section{REFERÊNCIAS}

1. Brasil. Lei nº 8.080, de 19 de setembro de 1990. Dispõe sobre as condições para a promoção, proteção e recuperação da saúde, a organização e o funcionamento dos serviços correspondentes e dá outras providências. Diário Oficial da União. Disponível em:

http://www.planalto.gov.br/ccivil_03/leis/18080.htm.

2. Silva MVS, Miranda GBN, Andrade MA. Diverse meanings of comprehensiveness:between the presupposed and the experienced in a multi-disciplinary team. Interface (Botucatu). 2017;21(62):589599. DOI: https://10.1590/1807-57622016.0420.

3. Ministério da Saúde, Secretaria de Atenção à Saúde, Departamento de Atenção Básica. Práticas em reabilitação na AB: o olhar para a funcionalidade na interação com o território 2017. Disponível em: http://bvsms.saude.gov.br/bvs/publicacoes/praticas_reabilitacao_atencao_basica_territorio.pdf.

4. Campos GWS. Saúde pública e saúde coletiva: campo e núcleo de saberes e práticas. Sociedade e Cultura, Jan-Dez. 2000;3(1-2):51-74. DOI: https://doi.org/10.1590/S1413-81232000000200002.

5. Ministério da Saúde, Centro de Estudo e Pesquisa em Saúde Coletiva. Guia prático de matriciamento em saúde mental. Brasília: DRQ Gráfica e Editora; 2011.

6. Réa-Neto A. Raciocínio clínico: o processo de decisão diagnóstica e terapêutica. Rev Ass Med Brasil. 1998;44(4):301-11. DOI: https://10.1590/S0104-42301998000400009.

7. World Health Organization. WHO. International Classification of functioning, disability and health. ICF 2003. Disponível em: https://apps.who.int/iris/bitstream/handle/10665/42407/9241545429.pdf.

8. Nubila HBV. Uma introdução à CIF: Classificação Internacional de Funcionalidade, Incapacidade e Saúde. Rev Bras Saúde Ocup. 2010;35(121):122-123. DOI: https://10.1590/S030376572010000100013.

9. Ministério da Saúde. Resolução No 510, de 7 de abril de 2016. Princípios Éticos. Disponível em: https://bvsms.saude.gov.br/bvs/saudelegis/cns/2016/res0510_07_04_2016.html.

10. Morán J. Mudando a educação com metodologias ativas. PROEX/UEPG 2015;2(1):15-33.

11. Universidade Federal de Pernambuco. Relatório Perfil Curricular 6804 - 1. Disponível em: https://www.ufpe.br/documents/39334/0/Perfil+Curricular+mar\%C3\%A7o+2021/848f86ca-67764227-86c0-eec40c6e3543.

12. Farias N, Buchalla CM. A Clasificação Internacional de Funcionalidade, Incapacidade e Saúde da Organização Mundial da Saúde: conceitos, usos e perspectivas. Rev Bras Epidemiol 2005;8:187-93.

13. Brisolla L. A prática pedagógica no ensino superior: planejamento, interdisciplinaridade e metodologias ativas. DED 2020;4(1):77-92. DOI: https://doi.org/10.30905/ded.v4il.157.

14. Diesel A, Baldez ALS, Martins SN. Os princípios das metodologias ativas de ensino: uma abordagem teórica. Rev Thema 2017;14(1):268-8. DOI: https://doi.org/10.15536/thema.14.2017.268-288.404.

15. Neto A, Moreira ÁRC, Pinheiro BE, Gomes JS, Otal LO, Fragoso MN. Quadro 5w2h: Uma ferramenta para definição do problema de projeto e de suas variáveis. Perspectivas Online: Humanas \& Sociais Aplicadas 2016;6(16):23-30. DOI: https://doi.org/10.25242/887661620161037. 
16. Joyce CR, Moreira MM, Rocha SSD. Educação a Distânci ou Atividade Educacional Remota Emergencial: em busca do elo perdido da educação escolar em tempos de COVID-19. RSD 2020;9(7):e521974299-e521974299. DOI: https://doi.org/10.33448/rsd-v9i7.4299.

17. Castaneda L, Bergmann A, Bahia L. A Clasificação Internacional de Funcionalidade, Incapacidade e Saúde: uma revisão sistemática de estudos observacionais. Rev Bras Epidemiol. 2019;17(2):437-51. DOI: https://dx.doi.org/10.1590/1809-4503201400020012.

18. Ceccim RB. Educação interprofissional para o desenvolvimento de competências colaborativas em saúde. In: Toassi RFC [organizadora]. Interprofissionalidade e formação na saúde: onde estamos?. Porto Alegre: Rede UNIDA; 2017:49-67. DOI: https://doi.org/10.1590/1807-57622018.0477.

19. Morin E. A cabeça bem-feita: repensar a reforma, reformar o pensamento 8 ed. Jacobina E, tradutor. Rio de Janeiro: Bertrand Brasil; 1921. 128p.

20. Santos C. A Construção Social do Conceito de Identidade Profissional. Interações SNM abr. 2005;5(8). Disponível em: https://interacoes-ismt.com/index.php/revista/article/view/145.

21. Nascimento MAV. Dimensões da identidade profissional docente na formação inicial. Revista portuguesa de pedagogia. 2007; Ciências Sociais [cerca de 13 telas]. Disponível em: https://digitalisdsp.uc.pt/bitstream/10316.2/4560/1/11\%20-

$\% 20$ Dimensoes $\% 20$ da $\% 20$ identidade $\% 20$ profissional $\% 20$ docente $\% 20$ na $\% 20$ formacao $\% 20$ inicial.pd $\mathrm{f} ? \ln =\mathrm{pt}-\mathrm{pt}$.

22. Sampaio RF, Mancini MC, Gonçalves GGP, Bittencourt NFN, Miranda AD, Fonseca ST. Aplicação da Classificação Internacional de Funcionalidade, Incapacidade e Saúde (CIF) na prática clínica do fisioterapeuta. Rev. bras. fisioter. 2005;9(2):129-13.

23. Peduzzi M. Educação interprofissional para o desenvolvimento de competências colaborativas em saúde. In: Toassi RFC [organizadora]. Interprofissionalidade e formação na saúde: onde estamos?. Porto Alegre: Rede UNIDA; 2017:40-48. 